\title{
Holomorphic 2-forms and Vanishing Theorems for Gromov-Witten Invariants
}

\author{
Junho Lee
}

\begin{abstract}
On a compact Kähler manifold $X$ with a holomorphic 2-form $\alpha$, there is an almost complex structure associated with $\alpha$. We show how this implies vanishing theorems for the Gromov-Witten invariants of $X$. This extends the approach, used in LP for Kähler surfaces, to higher dimensions.
\end{abstract}

Let $X$ be a Kähler surface with a non-zero holomorphic 2 -form $\alpha$. Then $\alpha$ is a section of the canonical bundle and its zero locus $Z_{\alpha}$, with multiplicity, is a canonical divisor. We showed in $\mathrm{L}$ that the real 2-form $\operatorname{Re}(\alpha)$ determines a (non-integrable) almost complex structure $J_{\alpha}$ that has the following remarkable "Image Localization Property" : if a $J_{\alpha}$-holomorphic map $f: C \rightarrow X$ represents a non-zero $(1,1)$ class, then $f$ is in fact holomorphic and its image lies in $Z_{\alpha}$. As shown in [LP], this property together with Gromov Convergence Theorem leads to:

Theorem 1 ([LP] $)$ Let $X$ be a Kähler surface with a non-zero holomorphic 2-form $\alpha$. Then, any class $A$ with non-trivial Gromov-Witten invariant $G W_{g, k}(X, A)$ is represented by a stable holomorphic map $f: C \rightarrow X$ whose image lies in the canonical divisor $Z_{\alpha}$.

This paper extends Theorem 1 to higher dimensions. The principle is the same: perturbing the Kähler structure to a non-integrable almost complex structure $J_{\alpha}$ forces the holomorphic maps to satisfy certain geometric conditions determined by $\alpha$. This gives constraints on the Gromov-Witten invariants.

Specifically, let $X$ be a compact Kähler manifold with a non-zero holomorphic 2-form $\alpha$. Then the real part of $\alpha$ defines an endomorphism $K_{\alpha}$ of $T X$ and an almost complex structure $J_{\alpha}$, just as in the surface case (see (2.1) and (2.2)). These geometric structures lead, naturally and easily, to our main theorem :

Theorem 2 Let $X$ be a compact Kähler manifold with a non-zero holomorphic 2-form $\alpha$. Then any class $A$ with non-trivial Gromov-Witten invariant $G W_{g, k}(X, A)$ is represented by a stable holomorphic map $f: C \rightarrow X$ satisfying the equation $K_{\alpha} d f=0$.

This theorem follows from Theorem 3.1] which is more suitable for applications. It generalizes Theorem 1 since when $X$ is a surface the kernel of the endomorphism $K_{\alpha}$ is trivial on $X \backslash Z_{\alpha}$ (see Example [3.5). The equation $K_{\alpha} d f=0$ is a geometric fact about holomorphic maps that directly implies numerous vanishing results about Gromov-Witten invariants (see Section 3). 
Section 1 briefly describes Gromov-Witten invariants and states a vanishing principle for them. Section 2 contains the definition of the almost complex structures $J_{\alpha}$ and some of the consequences of that definition. In Section 3 we apply a stronger version of Theorem 2, which directly follows from properties of $J_{\alpha}$, to show various vanishing results for Gromov-Witten invariants.

Acknowledgments. I am very grateful to T. Parker for useful discussions and encouraging conversations. I am also very thankful to the referee for several helpful comments and corrections.

\section{Gromov-Witten Invariants}

The aim of this section is to give a brief description of the Gromov-Witten invariants and to set up notations for them. Let $(X, \omega)$ be a compact symplectic $2 n$-dimensional manifold with an $\omega$-tamed almost complex structure $J$, i.e., $\omega(u, J u)>0$. A $J$-holomorphic map $f:(C, j) \rightarrow X$ from a (connected) marked nodal curve is stable if its automorphism group is finite (cf. [HZ). Denote by

$$
\overline{\mathcal{M}}_{g, k}(X, A, J)
$$

the moduli space of stable $J$-holomorphic maps from marked nodal curves of (arithmetic) genus $g$ with $k$ marked points that represent the homology class $A \in H_{2}(X)$. This moduli space carries a (virtual) fundamental homology class of real dimension

$$
2\left[c_{1}(T X) \cdot A+(n-3)(1-g)+k\right]
$$

(cf. [LT]) whose push-forward under the map

$$
s t \times e v: \overline{\mathcal{M}}_{g, k}(X, A, J) \rightarrow \overline{\mathcal{M}}_{g, k} \times X^{k}
$$

defined by stabilization and evaluation at the marked points is the Gromov-Witten invariant

$$
G W_{g, k}(X, A) \in H_{*}\left(\overline{\mathcal{M}}_{g, k} \times X^{k} ; \mathbb{Q}\right) .
$$

This is equivalent to the collection of "GW numbers"

$$
G W_{g, k}(X, A)\left(\mu ; \gamma_{1}, \ldots, \gamma_{k}\right)
$$

obtained by evaluating the homology class (1.2) on the cohomology classes Poincaré dual to $\mu \in H_{*}\left(\overline{\mathcal{M}}_{g, k}\right)$ and $\gamma_{j} \in H_{*}(X)$ whose total degree is the dimension (1.1). Standard cobordism arguments then show that these are independent of the choice of $J$, and depend only on the deformation class of the symplectic form $\omega$.

Our subsequent discussions are based on the following vanishing principle for GW invariants.

Proposition 1.1 Fix a compact symplectic manifold $(X, \omega)$. Suppose

$$
G W_{g, k}(X, A)\left(\mu ; \gamma_{1}, \cdots, \gamma_{k}\right) \neq 0 .
$$

Then, for any $\omega$-tamed almost complex structure $J$ and for any geometric representatives $M \subset$ $\overline{\mathcal{M}}_{g, k}$ and $\Gamma_{j} \subset X$ of classes $\mu \in H_{*}\left(\overline{\mathcal{M}}_{g, k}\right)$ and $\gamma_{j} \in H_{*}(X)$ there exists a stable $J$-holomorphic map $f:\left(C, x_{1}, \cdots, x_{k}\right) \rightarrow X$ representing class $A$ with $s t(C) \in M$ and $f\left(x_{j}\right) \in \Gamma_{j}$. 
The proof is straightforward (cf. $[\mathrm{LP}]$ ). For convenience, we will assemble all GW invariants for a class $A$ into a single invariant by introducing a variable $\lambda$ to keep track of the genus. The GW series of $(X, \omega)$ for a class $A$ is then the formal power series

$$
G W_{A}(X)=\sum_{g, k} \frac{1}{k !} G W_{g, k}(X, A) \lambda^{g} .
$$

\section{The Almost Complex Structures $J_{\alpha}$}

Let $(X, \omega)$ be a compact symplectic manifold with an $\omega$-compatible almost complex structure $J$, namely $\langle u, v\rangle=\omega(u, J v)$ is a Riemannian metric. A 2 -form $\alpha$ is then called $J$-anti-invariant if $\alpha(J u, J v)=-\alpha(u, v)$. As observed in $\underline{\mathrm{L}}$, each $J$-anti-invariant 2 -form $\alpha$ induces an almost complex structure

$$
J_{\alpha}=\left(I d+J K_{\alpha}\right)^{-1} J\left(I d+J K_{\alpha}\right)
$$

where $K_{\alpha}$ is an endomorphism of $T X$ defined by the equation

$$
\left\langle u, K_{\alpha} v\right\rangle=\alpha(u, v)
$$

Such endomorphisms $K_{\alpha}$ are skew-adjoint and anti-commute with $J$. It follows that $I d+J K_{\alpha}$ is invertible and hence (2.1) is well-defined. A simple computation then shows that for any $C^{1}$ map $f:(C, j) \rightarrow X$,

$$
\bar{\partial}_{J_{a}} f=0 \quad \Longleftrightarrow \quad \bar{\partial}_{J} f=K_{\alpha} \partial_{J} f j
$$

where

$$
\bar{\partial}_{J} f=\frac{1}{2}(d f+J d f), \quad \partial_{J} f=\frac{1}{2}(d f-J d f j) .
$$

(2.3) implies that every $J$-holomorphic map $f$ satisfying $K_{\alpha} d f=0$ is also $J_{\alpha}$-holomorphic. One can also show that if $f$ is $J_{\alpha}$-holomorphic then

$$
\int_{C}\left|\bar{\partial}_{J} f\right|^{2}=\int_{C}\left|K_{\alpha} \partial_{J} f\right|^{2}=\int_{C} f^{*}(\alpha)
$$

(cf. $\mathrm{L}$ ). This integral vanishes when $\alpha$ is closed and $\alpha(A)=0$ where $A$ is the class represented by $f$. In this case, the given $J_{\alpha}$-holomorphic map $f$ is $J$-holomorphic $\left(\bar{\partial}_{J} f=0\right)$ and satisfies $K_{\alpha} d f=K_{\alpha} \partial_{J} f=0$. Therefore, when $\alpha$ is closed and $\alpha(A)=0$, a map $f$ representing the class $A$ is $J_{\alpha}$-holomorphic if and only if $f$ is $J$-holomorphic and satisfies the equation $K_{\alpha} d f=0$. Combined with Proposition 1.1, these observations lead to:

Proposition 2.1 Let $(X, \omega)$ be a compact symplectic manifold with an $\omega$-compatible $J$ and with a closed J-anti-invariant 2-form $\alpha$. Then, for any class $A$ with $G W_{g, k}(X, A) \neq 0$ we have

$$
\overline{\mathcal{M}}_{g, k}\left(X, A, J_{\alpha}\right)=\left\{f \in \overline{\mathcal{M}}_{g, k}(X, A, J) \mid K_{\alpha} d f=0\right\} .
$$

In particular, this space is not empty. 
Proof. By the above discussion, it suffices to show that $\alpha(A)=0$ and $\overline{\mathcal{M}}_{g, k}\left(X, A, J_{\alpha}\right) \neq \emptyset$. Proposition 1.1] shows that there is a $J$-holomorphic map $h:(D, j) \rightarrow X$ representing the class $A$. Fix a point $p \in D$ and choose an orthonormal basis $\left\{e_{1}, e_{2}=j e_{1}\right\}$ of $T_{p} D$. Then,

$$
h^{*} \alpha\left(e_{1}, e_{2}\right)=\alpha\left(h_{*} e_{1}, h_{*} j e_{1}\right)=\alpha\left(h_{*} e_{1}, J h_{*} e_{1}\right) .
$$

Since $\alpha$ is $J$-anti-invariant, this vanishes and hence $\alpha(A)=\int_{D} h^{*}(\alpha)=0$. On the other hand, for any sufficiently small $t>0$ the almost complex structure $J_{t \alpha}$ is $\omega$-tamed since $\omega$-tamed is an open condition. Proposition 1.1 then asserts that there exists a $J_{t \alpha}$-holomorphic map $f$ representing the class $A$. By (2.4) and the fact $K_{t \alpha}=t K_{\alpha}$, this map $f$ is $J$-holomorphic and satisfies $K_{\alpha} d f=0$. Thus, $f$ is also $J_{\alpha}$-holomorphic by (2.3).

Below, we will show some basic properties of the zero locus $Z_{\alpha}$ of $\alpha$ and ker $K_{\alpha}$, which will be frequently used in subsequent arguments. One can use $J$ to decompose $\Omega^{2}(X) \otimes \mathbb{C}$ as

$$
\Omega^{2}(X) \otimes \mathbb{C}=\Omega_{J}^{2,0}(X) \oplus \Omega_{J}^{1,1}(X) \oplus \Omega_{J}^{0,2}(X) .
$$

Every $J$-anti-invariant 2 -form $\alpha$ then can be written as $\alpha=\beta+\bar{\beta}$ for some $\beta \in \Omega_{J}^{2,0}(X)$. The following lemma simply follows from the definitions and the properties of $K_{\alpha}$.

Lemma 2.2 Let $\operatorname{dim} X=2 n$, and $\alpha$ and $\beta$ be as above. Then,

(a) $\alpha$ and $\beta$ have the same zero locus,

(b) if $n$ is odd then $\alpha^{n}=0$, and if $n=2 m$ then $\alpha^{n}=c \beta^{m} \wedge \bar{\beta}^{m}$ where $c=\left(\begin{array}{c}n \\ m\end{array}\right)$,

(c) the (real) dimension of ker $K_{\alpha}$ is at most $2 n-4$ at every point in $X \backslash Z_{\alpha}$,

(d) $u \in \operatorname{ker} K_{\alpha}$ if and only if $\alpha(u, w)=0$ for any $w$. Thus, $\operatorname{ker} K_{\alpha}$ is trivial if and only if $\alpha$ is non-degenerate.

A foliation $\mathcal{F}$ of dimension $m$ on $n$-dimensional manifold $M$ is a decomposition $\mathcal{F}=\left(L_{i}\right)_{i \in I}$ of $M$ into pairwise disjoint connected subsets $L_{i}$, which are called leaves of the foliation $\mathcal{F}$, with the following property: for each $p \in M$ there exists a foliation chart $\varphi: U \rightarrow W_{1} \times W_{2}$, where $W_{1}$ and $W_{2}$ are open disks in $\mathbb{R}^{m}$ and $\mathbb{R}^{n-m}$ respectively, such that for each point $q \in W_{2}$ the preimage $\varphi^{-1}\left(W_{1} \times\{q\}\right)$ is a connected component of $U \cap L_{i}$ for some leaf $L_{i}$. We refer to CN] and $\underline{\mathrm{Ho}}$ for more details on foliations.

Lemma 2.3 Let $(X, \omega)$ be a six dimensional symplectic manifold with $\omega$-compatible $J$. If $\alpha$ is a closed $J$-anti-invariant 2-form, then ker $K_{\alpha}$ gives a foliation on $X \backslash Z_{\alpha}$ of (real) dimension two whose leaves are all J-invariant.

Proof. Since $K_{\alpha}$ is anti-commute with $J$, Lemma 2.2] implies that on $X \backslash Z_{\alpha}$ the dimension of ker $K_{\alpha}$ is two. On the other hand, $d \alpha(u, v, w)=0$ gives

$$
L_{u}(\alpha(v, w))-L_{v}(\alpha(u, w))+L_{w}(\alpha(u, v))-\alpha([u, v], w)+\alpha([u, w], v)-\alpha([v, w], u)=0
$$

where $L$ denotes the Lie derivative. This together with Lemma 2.2 d imply that if $u, v \in$ ker $K_{\alpha}$ then $[u, v] \in \operatorname{ker} K_{\alpha}$. Therefore, by Frobenius Theorem ker $K_{\alpha}$ gives a foliation on $X \backslash Z_{\alpha}$ of dimension two. Since $K_{\alpha}$ is anti-commute with $J$, every leaf is $J$-invariant. 


\section{Vanishing Results}

Let $(X, J)$ be a compact Kähler manifold with a non-zero holomorphic 2-form $\alpha$. By the Hodge Theorem $\alpha$ is closed and hence its real part $\operatorname{Re}(\alpha)$ is also closed. Moreover, the real 2-form $\operatorname{Re}(\alpha)$ is $J$-anti-invariant and its zero locus is $Z_{\alpha}$ by Lemma 2.2 a. Throughout this section, we will denote by $K_{\alpha}$ the endomorphism of $T X$ defined by $\operatorname{Re}(\alpha)$ as in (2.2).

A holomorphic 2-form $\alpha$ is called non-degenerate if $\operatorname{Re}(\alpha)$ is non-degenerate, or equivalently ker $K_{\alpha}$ is trivial. The following theorem directly follows from Proposition 1.1 and Proposition 2.1

Theorem 3.1 Fix a compact Kähler manifold $X$ with a non-zero holomorphic 2-form $\alpha$. If for a non-zero class $A$

$$
G W_{g, k}(X, A)\left(\mu ; \gamma_{1}, \cdots, \gamma_{k}\right) \neq 0
$$

then for any geometric representatives $M \subset \overline{\mathcal{M}}_{g, k}$ and $\Gamma_{j} \subset X$ of classes $\mu \in H_{*}\left(\overline{\mathcal{M}}_{g, k}\right)$ and $\gamma_{j} \in H_{*}(X)$ there exists a stable holomorphic map $f:\left(C, x_{1}, \cdots, x_{k}\right) \rightarrow X$ representing the class $A$ with st $(C) \in M$ and $f\left(x_{j}\right) \in \Gamma_{j}$ and satisfying the equation $K_{\alpha} d f=0$. Consequently, if $\alpha$ is non-degenerate on an open set $U \subset X$ then the image of $f$ lies in $X \backslash U$.

Using this Theorem, one can obtain various vanishing results about GW invariants.

Example 3.2 Given a compact hyperkähler manifold $X$ of (complex) dimension $2 m$, there exists a holomorphic symplectic 2 -form $\alpha$, i.e. $\alpha^{m}$ is nowhere vanishing (cf. BDL]). The 2-form $\alpha$ is non-degenerate on $X$ and hence Theorem 3.1 implies that the series $G W_{A}(X)$ vanishes unless $A=0$.

Example 3.3 Let $X=E_{1} \times \cdots \times E_{n}$ where each $E_{i}$ is an elliptic curve and $n \geq 2$. For $i \neq j$, denote by $\alpha_{i j}$ the pull-back 2 -form $\pi_{i}^{*}\left(\lambda_{i}\right) \wedge \pi_{j}^{*}\left(\lambda_{j}\right)$ where $\pi_{i}: X \rightarrow E_{i}$ is the $i$-th projection and $\lambda_{i}$ is a nowhere vanishing holomorphic 1-form on $E_{i}$. Now, suppose $G W_{A}(X) \neq 0$. Theorem 3.1 then shows that there is a holomorphic map $f: C \rightarrow X$ representing the class $A$ with $K_{\alpha_{i j}} d f=0$. Since $\alpha_{i j}$ has no zeros and ker $K_{\alpha_{i j}}$ consists of vectors tangent to fibers of the projection $\pi_{i} \times \pi_{j}: X \rightarrow E_{i} \times E_{j}$, we have $\left(\pi_{i} \times \pi_{j}\right)_{*} d f=0$ for each $i \neq j$. This implies $A=0$. The same arguments also apply to show that when $X=X_{1} \times \cdots \times X_{n}$ where each $X_{i}$ is a hyperkähler manifold or a complex torus of (complex) dimension at least two the series $G W_{A}(X)$ vanishes unless $A=0$.

Remark 3.4 There are well-known proofs for the above two examples (cf. BL]). For instance, if $X$ is a compact hyperkähler manifold then every Kähler structure $J$ in the twistor family is deformation equivalent to $-J$ through Kähler structures (cf. BDL). This directly implies $G W_{A}(X)=0$ unless $A=0$. The product formula of $[\mathrm{B}$ for $\mathrm{GW}$ invariants applies to give the same vanishing results as in Example 3.3 .

The following example appears in $[\mathrm{LP}$.

Example 3.5 ([LP]) Let $X$ be a Kähler surface with a non-zero holomorphic 2-form $\alpha$. Then, $\alpha$ is non-degenerate on $X \backslash Z_{\alpha}$ by Lemma $2.2 \mathrm{c}$,d. Note that since $\alpha$ is a section of the canonical 
bundle the zero locus $Z_{\alpha}$ is a support of a canonical divisor. Theorem 3.1 thus shows that for any non-zero class $A$ and for any genus $g$

$$
G W_{g, k}(X, A)(\cdot ; \gamma, \cdots)=0
$$

where $\gamma$ lies in $H_{i}(X)$ for $i=0,1$. On the other hand, if $X$ is minimal surface of general type then every canonical divisor is connected (cf. [BHPV] $)$. We further assume that the zero locus $Z_{\alpha}$ is a smooth (reduced) canonical divisor. Then, any holomorphic map $f$ whose image lies in $Z_{\alpha}$ represents a (non-negative) multiple of the canonical class $K$. Therefore, Theorem 3.1 implies that the series $G W_{A}(X)$ vanishes unless $A=m K$ for some non-negative integer $m$.

The following example extends both the vanishing result (3.1) and Theorem 1 of the introduction to Kähler manifolds of even complex dimension. It is an immediate consequence of Theorem 3.1 .

Example 3.6 Fix a compact Kähler manifold $X$ of complex dimension $2 m$ with a holomorphic 2 -form $\alpha$. If $\alpha^{m}$ is not identically zero, then the zero locus $Z_{m}$ of $\alpha^{m}$, with multiplicities, is a canonical divisor of $X$ and $\alpha$ is non-degenerate on $X \backslash Z_{m}$. Theorem 3.1 implies that:

(a) if $G W_{g, k}(X, A) \neq 0$ for a non-zero class $A$, then $A$ is represented by a stable holomorphic map $f: C \rightarrow X$ whose image lies in the canonical divisor $Z_{m}$, and

(b) for any non-zero class $A$ and for any genus $g$ we have $G W_{g, k}(X, A)(\cdot ; \gamma, \cdots)=0$ where $\gamma$ lies in $H_{i}(X)$ for $i=0,1$.

\section{Compact Kähler Threefolds}

Let $X$ be a compact Kähler threefold with a non-zero holomorphic 2-form $\alpha$. It then follows from Lemma 2.3 that ker $K_{\alpha}$ induces a foliation on $X \backslash Z_{\alpha}$ of (real) dimension two. We will denote this foliation by $\mathcal{F}_{\alpha}$.

Lemma 3.7 Fix a compact Kähler threefold $X$ with a non-zero holomorphic 2-form $\alpha$. If a (non-constant) stable holomorphic map $f: C \rightarrow X$ satisfies the equation $K_{\alpha} d f=0$, then

- the image of each irreducible component of $C$ either lies in $Z_{\alpha}$ or lies in one leaf of the foliation $\mathcal{F}_{\alpha}$ on $X \backslash Z_{\alpha}$ union finitely many points of $Z_{\alpha}$.

Consequently, if $\alpha$ has no zeros then the image of $f$ lies in one leaf of the foliation $\mathcal{F}_{\alpha}$ on $X$.

Proof. Collapse all irreducible components of $C$ whose image is a point. The resulting map still has the same image $f(C)$, so we can assume that the image of each irreducible component is not a point. Fix an irreducible component $C_{i}$ of $C$ and suppose $f\left(C_{i}\right)$ is not contained in $Z_{\alpha}$. Then, the intersection $f\left(C_{i}\right) \cap Z_{\alpha}$ is finite since $f$ is holomorphic and $Z_{\alpha}$ is an analytic subvariety. Denote by $D_{i}$ the set of critical points of $f$ in $C_{i}$. This set $D_{i}$ is finite and hence $C_{i} \backslash\left(D_{i} \cup f^{-1}\left(Z_{\alpha}\right)\right)$ is open and connected. Therefore, the equation $K_{\alpha} d f=0$ asserts that $f\left(C_{i} \backslash D_{i}\right) \backslash Z_{\alpha} \subset L_{i}$ for some leaf $L_{i}$ of the foliation $\mathcal{F}_{\alpha}$ on $X \backslash Z_{\alpha}$. It then remains to show that for each $p \in D_{i}$ either $f(p) \in Z_{\alpha}$ or $f(p) \in L_{i}$. Suppose $f(p)$ does not lie in $Z_{\alpha}$. Let $(U, \varphi)$ be a foliation chart around $f(p)$, namely $U \subset X \backslash Z_{\alpha}$ is a neighborhood of $f(p)$ and 
$\varphi(U)=W_{1} \times W_{2} \subset \mathbb{R}^{2} \times \mathbb{R}^{4}$, where $W_{1}$ and $W_{2}$ are open disks in $\mathbb{R}^{2}$ and $\mathbb{R}^{4}$ respectively, such that for each point $t \in W_{2}$ the pre-image $\varphi^{-1}\left(W_{1} \times\{t\}\right)$ is a connected component of $U \cap L_{t}$ for some leaf $L_{t}$ of $\mathcal{F}_{\alpha}$. Then, for any small neighborhood $V \subset C_{i}$ of $p$ there exists a point $t_{i} \in W_{2}$ such that $\varphi \circ f(V \backslash\{p\}) \subset W_{1} \times\left\{t_{i}\right\}$. Consequently, we have $\varphi \circ f(p) \in W_{1} \times\left\{t_{i}\right\}$. Since the pre-image $\varphi^{-1}\left(W_{1} \times\left\{t_{i}\right\}\right)$ is a connected component of $U \cap L_{i}$, we have $f(p) \in L_{i}$.

Example 3.8 Fix a surface of general type $S$ with a holomorphic 2-form $\gamma$ whose zero locus is a smooth canonical divisor $D$. Let $\pi: X=\mathbb{P}(T S) \rightarrow S$ be the projective bundle with a pull-back 2-form $\alpha=\pi^{*} \gamma$. The zero locus $Z_{\alpha}$ is then the ruled surface $\pi^{-1}(D) \rightarrow D$ and every leave of the foliation $\mathcal{F}_{\alpha}$ on $X \backslash \pi^{-1}(D)$ is a fiber of $\pi: X \rightarrow S$. Thus, Theorem 3.1 and Lemma 3.7 together imply that $G W_{A}(X)=0$ unless $A=a D_{0}+b F$ for some integers $a$ and $b$ where $D_{0}$ is the section class of the ruled surface $\pi^{-1}(D)$ and $F$ is the fiber class of $X$.

Now, suppose $X$ is a compact threefold with a holomorphic 2-form $\alpha$ without zeros. The foliation $\mathcal{F}_{\alpha}$ is then a foliation on the whole $X$. In fact, $\mathcal{F}_{\alpha}$ is a holomorphic foliation; in a holomorphic local coordinates, the foliation $\mathcal{F}_{\alpha}$ is given locally by the holomorphic vector field

$$
Y=f_{23} \frac{\partial}{\partial z_{1}}-f_{13} \frac{\partial}{\partial z_{2}}+f_{12} \frac{\partial}{\partial z_{3}}
$$

where $\alpha=f_{12} d z_{1} \wedge d z_{2}+f_{13} d z_{1} \wedge d z_{3}+f_{23} d z_{2} \wedge d z_{3}$. After suitable change of local coordinates, we can write $Y=\frac{\partial}{\partial z_{1}}$. Such local coordinates give the required holomorphic foliation chart.

Corollary 3.9 Let $X$ be a compact Kähler threefold with a holomorphic 2-form $\alpha$ without zeros. Suppose $X$ is not a $\mathbb{P}^{1}$-bundle over a $K 3$ or an abelian surface. Then for any non-zero class $A$ the invariant

$$
G W_{g, k}(X, A)(\cdot ; \gamma, \cdots)
$$

vanishes if the genus $g$ is 0 or if one constraint $\gamma$ lies in $H_{i}(X)$ for $0 \leq i \leq 3$.

Proof. Assume that for some $A \neq 0$ the invariant (3.2) is not zero with either $g=0$ or $\gamma \in H_{i}(X)$ for $0 \leq i \leq 3$. We will show that $X$ is a $\mathbb{P}^{1}$-bundle over a $\mathrm{K} 3$ or an abelian surface. Since every leaf of $\mathcal{F}_{\alpha}$ is a smooth connected holomorphic curve, by Theorem 3.1 and Lemma 3.7 there exists a stable holomorphic map $f: C \rightarrow X$ representing the non-zero class $A$ with $f(C)=L$ for some leaf $L$ of $\mathcal{F}_{\alpha}$. The leaf $L$ is thus compact and $A=m[L]$ for some integer $m \geq 1$. If the genus $g$ is 0 then obviously $L=\mathbb{P}^{1}$.

On the other hand, if $\gamma$ lies in $H_{i}(X)$ for $0 \leq i \leq 3$ and the invariant (3.2) is non-zero, then the formal dimension (1.1) of the moduli space $\mathcal{M}_{g, 0}(X, A)$ is strictly positive, so $c_{1}(X) \cdot A \geq 2$. But by Theorem 2 of $\left[\mathrm{Le}\right.$, the normal bundle $N$ to $L$ satisfies $c_{1}(N)=0$, so

$$
2 \leq c_{1}(X) A=m c_{1}(X)[L]=m\left(c_{1}(L)+c_{1}(N)\right)[L]=m c_{1}(L) .
$$

Hence $c_{1}(L)=2$ and therefore $L=\mathbb{P}^{1}$ in this case also.

Now, by the proof of Corollary 2.8 of $[\underline{\mathrm{H}}]$, the fact that one leaf $L$ of $\mathcal{F}_{\alpha}$ is a rational curve $\mathbb{P}^{1}$ implies that every leaf of $\mathcal{F}_{\alpha}$ is rational. It follows that the leaf space $S=X / \mathcal{F}_{\alpha}$ is a (smooth) compact Kähler surface and the quotient map $\pi: X \rightarrow S$ is holomorphic. Consequently, $X$ is a $\mathbb{P}^{1}$-bundle over $S$ and $\alpha$ descends to a holomorphic 2-form $\gamma$ on $S$ with $\pi^{*} \gamma=\alpha$. Since the holomorphic 2-form $\gamma$ has no zeros, $c_{1}(S)=0$ and hence $S$ is a K3 or an abelian surface (cf. [BHPV]). 
Remark 3.10 Let $X$ be a projective threefold with a holomorphic 2-form $\alpha$. Suppose that for some non-zero class $A$ the invariant $G W_{g, k}(X, A)(\cdot ; \gamma, \cdots)$ with the constraint $\gamma \in H_{i}(X)$ for $0 \leq i \leq 3$ does not vanish. Then the canonical divisor $K_{X}$ is not nef by a dimension count. In this case, there is a contraction $\pi: X \rightarrow S$ of an extremal ray such that $X$ is a $\mathbb{P}^{1}$-bundle over a surface $S$ and $\alpha$ descends to a holomorphic 2 -form $\gamma$ on $S$ with $\pi^{*} \gamma=\alpha$ (see Section 3 of [CP]). Consequently, if $\alpha$ has isolated zeros then $S$ is a K3 or an abelian surface and, in fact, $\alpha$ has no zeros. This observation motivated Corollary 3.9

\section{References}

[B] K. Behrend, The product formula for Gromov-Witten invariants J. Algebraic Geom. 8 (1999), no. 3, $529-541$.

[BDL] J. Bryan, R. Donagi, N.C. Leung, G-bundles on abelian surfaces, hyperkahler manifolds, and stringy Hodge numbers. Turkish J. Math. 25 (2001), no. 1, 195-236.

[BHPV] W. Barth, K. Hulek, C. Peters, and A. Van de Ven, Compact complex surfaces, second ed., Springer-Verlag, Berlin Heidelberg, 2003.

[BL] J. Bryan and N. C. Leung, Counting curves on irrational surfaces, Surveys in differential geometry: differential geometry inspired by string theory, 313-339, Surv. Diff. Geom., 5, Int. Press, Boston, MA, 1999.

[CN] C. Camacho and A.L. Neto, Geometric Theory of Foliations, Translated from the Portuguese by Sue E. Goodman. Birkhuser Boston, Inc., Boston, MA, 1985.

[CP] F. Campane and T. Peternell, Complex threefolds with non-trivial holomorphic 2-forms, J. Algebraic Geom. 9 (2000), no. 2, 223-264.

[H] A. Hoering, Uniruled varieties with splitting tangent bundle, preprint, math.AG/0505327

[Ho] H. Holmann, On the stability of holomorphic foliations, Analytic functions, Kozubnik 1979 (Proc. Seventh Conf., Kozubnik, 1979), pp. 192-202, Lecture Notes in Math., 798, Springer, Berlin, 1980.

[HZ] K. Hori, S. Katz, A. Klemm, R. Pandharipande, R. Thomas, C. Vafa, R. Vakil, and E. Zaslow, Mirror symmetry, Clay Mathematics Monographs, 1. American Mathematical Society, Providence, RI, Clay Mathematics Institute, Cambridge, MA, 2003.

[L] J. Lee, Family Gromov-Witten Invariants for Kähler Surfaces, Duke Math. J. 123 (2004), 209-233.

[LP] J. Lee and T. H. Parker, A structure theorem for the Gromov-Witten invariants of Kähler surfaces, to appear in J. Diff. Geom, preprint math.SG/0610570

[Le] D. Lehmann, Résidues des sous-variétés invariantes d'un feuilletage singulier, Ann. Inst. Fourier 41 (1991), 211-258.

[LT] J. Li and G. Tian, Virtual moduli cycles and Gromov-Witten invariants of general symplectic manifolds, Topics in symplectic 4-manifolds (Irvine, CA, 1996), 47-83, First Int. Press Lect. Ser. I, International Press, Cambridge, MA, 1998.

Mathematics Department, Michigan State University, East Lansing, MI 48824

E-mail addresses: leejunho@msu.edu 